(Aus der Station für Ohrenkranke des Garnison-Lazaretts I StraBburg i. Els.)

\title{
Zur Klinik des Herpes zoster oticus.
}

\author{
Von \\ Dr. Arthur Jaehne, \\ Königl. Sächs. Stabsarzt.
}

Ein Herpes zoster an der Ohrmuschel im Ausbreitungsgebiet des N. auriculo-temporalis, eines Astes des N. trigeminus, wird nicht häufig beobachtet, seltener noch ist die Kombination dieser Erkrankung mit einer Affektion des $\mathrm{N}$. facialis und N. acusticus. Im ganzen sind etwa 15 derartige Fälle beschrieben worden; Körner (1) hat 1904 dieses Krankheitsbild analog dem seit längerer Zeit gut eingebürgerten Namen Herpes zoster ophthalmicus als Herpes zoster oticus bezeichnet. Bei jedem Herpes zoster erkrankt primär der Nerv' oder ein in seinem Verlauf eingeschaltetes Ganglion, die Zostereruption ist nach NeiBer (2) durch eine mehr oder weniger ausgedehnte Gangrän der Haut bedingt, und die Ursache dieser Gangrän ist höchstwahrscheinlich in dem Fehlen gewisser für die Ernährung der betreffenden Teile notwendigen Nervenimpulse zu suchen. Von dem gewöhnlichen Herpes zoster unterscheidet sich ein einfacher Herpes der Ohrmuschel nicht, wohl aber nimmt der sog. Herpes zoster oticus insofern eine Sonderstellung ein, als er mit einer Erkrankung eines motorischen und eines Sinnesnerven einhergeht, während der Herpes zoster im allgemeinen nur im Ausbreitungsgebiete sensibler Nerven auftritt. Lähmungen motorischer Nerven sind allerdings auch sonst bei Herpes zoster beobachtet worden, diese Fälle sind aber recht selten, sie betrafen ganz vereinzelt Nerven des Rumpfes und der Extremitäten, mehrmals den N. facialis bei Zostereruptionen im Trigeminusgebiet. Aus den Mitteilungen über Herpes 
zoster oticus, von denen einige freilich mit einem mehrere Wochen nach Beginn der Erkrankung aufgenommenen Endstatus abschließen, geht hervor, daß nach ursprünglicher Taubheit und kompleter Lähmung des Gesichtsfazialis zwar öfters dauernde Schädigungen des Gehörs nach der Erkrankung zurückgeblieben sind, auch eine teilweise Lähmung des. Gesichtsfazialis" weiter bestanden hat, eine bleibende völlige Taubheit und absolute vestibuläre Unerregbarkeit ist jedoch, soviel ich aus der Literatur ersehe, noch nicht beschrieben worden. Über einen derartig schwer verlaufenen Fall von Herpes zoster oticus zu beriehten, bin ich in der Lage:

Krankengeschich te.

20 jähriger Dragoner F. Ein Bruder lungenkrank, von 15 Gesehwistern 8 in Kindheit gestorben. Er selbst fruher stets gesund. Seit Anfang November 1911 ohne erkennbare Ursache Schluckbeschwerden, Mattigkeit, Appetitlosigkeit, allmählig stärker werdende Gelbfärbung der Haut, seit 9. November Gesichtsreißen rechts, besonders in der Stirngegend, und Taubheitsgefühl in rechter Gesichtshälfte. Krankmeldung am 11. 11. 11; am gleichen Tage Aufnahme im Garnisonlazarett Hagenau.

Status. 11. XI. 1911. Mittelgroßer, schlanker Mann in mittelgutem Ernährungszustande. Haut und Augenbindehäute ziemlich stark ikterisch verfärbt. Rötung der Gaumenmandeln, keine Beläge. Brustorgane regelrecht, Leib weich, nirgends druckschmerzhaft. Urin dunkelbraun, enthält Gallenfarbstoff. Stuhl tonfarben. Temperatur 37,5. Rehandlung: Fleisohlose Kost, Rad. Rhei.

12. XI. Schmerzen im rechten Ohr, Trommelfell o. B.

13. XI. In der rechten Ohrmuschel in der Konkavität mehrere linsengroße mit wasserklarem Inhalt gefullte und dünner Epidermis bedeckte Bläschen, auf gerötetem geschwollenen Grund, ebensolche im Gehörgange bis an Trommelfellrand reichend (Herpes zoster). Trommelfell etwas gerötet, nicht vorgewölbt. Hochnormale Temperatur.

15. XI. In der Nacht verspürte Patient ganz plötzlich ein heftiges Reißen in der rechten Gesichtshälfte. Am Morgen starke Schmerzen im rechten Ohr. Aufrichten nicht möglich, da der Kopf schwer, wie Blei niedergezogen wurde; beim Versuche, sich aufzurichten, Kopfschmerz, Brechreiz, subjektiver Drehschwindel. Die Gegenstände im Zimmer, das Bett, schienen sich zu drehen. Die rechte Gesichtshälfte ist vollkommen gelähmt. Rechtes Ohr: Sekretansammlung im äußeren. Gehörgang, normale Einziehung des Trommelfelles ausgeglichen. Lymphdrüsen in der Umgebung des rechten Ohres gering geschwollen. Flüstersprache dicht am Ohr nicht gehört.

In den nächsten Tagen völlige Entfieberung. Schwinden der Allgemeinsymptome. Gelbfärbung der Haut sich allmählich verlierend, Urin hell, ohne Gallenfarbstoff. Herpes an Ohrmuschel und Gehörgang heilt ab. Fazialislähmung unverändert. Am rechten $\mathrm{Ohr}$ nur laute Sprache gehört, Schwindel geringer; beim Aufstehen Umfallen nach rechts.

6. XII. Trommelfell von regelrechter Farbe und Spannung. Gehörgangswandungen glatt. Flüstersprache am Ohr nicht verstanden. Nur noch beim Bücken leichter Schwindel. Gang etwas schwankend.

16. XIr. Verlegung in Garnisonlazarett I Straßburg i. Els. zur spezialistischen Weiterbehandlung. 
17. XII. Befund der Ohrenstation des Garnisonlazaretts Straßburg: An rechter Ohrmuschel und Umgebung einige runde stecknadelkopf- bis hirsekorngroße, blaßrot gefärbte Hautstellen, kleine Narben. Trommelfell leicht eingezogen, sonst regelrecht. Hörprüfung: Weber nach links lateralisiert. Durchhören der auf $\boldsymbol{r}$. Warzenfortsatz anfgesetzten A-Gabel nach links bei starkem Anschlag. Rechts durch Luftleitung kein Hörvermögen für Stimmgabeltöne. Stimmgabeln von $c 2$ der kleingestrichenen Oktaven aufwärts nach links durchgehört, ebenso gedakte Pfeifen bei VerschluB beider Ohren gehört. Links Flüstersprache $25 \mathrm{~m}$ gehört. Kalorischer Nystagmus rechts nicht auslösbar, links regelrecht. Beim Fublidschluß Umfallen nach rechts.

Behandlung: Schwitzkur, Aspirin, Elektrisieren.

2. II. Befund im wesentlichen unverändert. Wassermannsche Reaktion negativ. Behandlung weiter mit Glektrisieren, Sand-Glühlichtbäder.

8. IV. Fazialislähmung und Taubheit auf dem rechten Ohr im wesentlichen unverändert. Klagen über Empfindlichkeit der rechten Gesichtshälfte gegen Berührungen und kaltes Wasser. In der letzten Zeit häufiger etwa eine Stunde nach dem Aufstehen Brechreiz. Rechtes Auge tränt angeblich stark. Beim Bücken und schnellen Umdrehen Schwindelgefübl, es wird dem Patienten dabei schwarz vor den Augen, und er bekommt eine Schwere in den Kopf. Die Austrittsstellen der 3 Trigeminusäste sind rechts stark druckempfindlich. Ein weiterer Druckpunkt hinter dem rechten Ohr. Beim FuBlidschluB Umfallen nach rechts.

17. VI. 13. Vollständige Lähmung des rechten Mundfazialis, AugenschlieBen gelingt rechts bis auf einen etwa $2 \mathrm{~mm}$ weiten Spalt, dabei weicht Augapfel nach oben ab. Keine elektrische Entartungsreaktion der Fazialis. Bei starker Reizung des Stirnfazialis zucken die übrigen Äste mit; bei starker faradischer Reizung krampfhaftes Verziehen der rechten Gesichtshälfte. Hörvermögen für Stimmgabeln und Sprache rechts völlig aufgehoben. Hörprüfungsbefund wie bei der ersten otologischen Untersuchung am 17. XII. Vestibularapparat rechts kalorisch völlig unerregbar. Beim Drehen nach links 5-6 Nachnystagmuszuckungen nach rechts, beim Drehen nach rechts $15-20$ Nachnystagmuszuckungen nach links. Links durchaus regelrechtes Hörvermögen für Stimmgabeltöne und Sprache; Vestibularapparat regelrecht erregbar. Bei Fußlidschlub leichtes Schwanken nach rechts. Austrittsstellen der drei Trigeminusäste druckschmerzhaft. Nadelstiche und Berührungen im Hautgebiet des Trigeminus ebenso deutlich wie links gespürt. Bei Geschmacksprüfung am Rand der vorderen Zungenhälfte rechts salzig, sauer, süb, bitter durchweg nicht geschmeckt, sondern nur als kalt bezeichnet, links regelrechte Ge schmacksenapfindung. - Tapfchen steht schief nach links. Gaumenbogen rechts tiefer als links.

Entlassung als dienstunbrauchbar wegen Gesichtsnervenlähmung und Taubheit rechts. Am 27. XI. 1913 berichtet der Patient, der wie vor der Dienstzeit als Bauarbeiter arbeitet, auf Anfrage, daB seine Taubheit und Gesichtslähmung unverändert wie bei der Entlassung vom Militär weiter bestehe.

Zusammenfassung: 21 jähriger früher gesunder Mann erkrankt unter den Zeichen einer Allgemeininfektion (Fieber, Abgeschlagenheit, Ikterus). Nach einigen Tagen tritt ein Herpes zoster an der Ohrmuschel und im Gehörgang auf; drei Tage später Fazialislähmung und schwerste Alterations- 
symptome seitens des Vestibularis- und Kochlearisteiles vom N. acusticus. In kurzer Zeit Taubheit und völlige Unerregbarkeit des Vestibularapparates eintretend. Beides bleibt dauernd bestehen, ebenso Geschmacksstörungen an rechter Zungenhälfte, während Fazialislähmung nach einem halben Jahr im Stirnast etwas gebessert ist.

Der Herpes zoster oticus war in einigen der beschriebenen Fälle im Anschluß an eine starke Abkühlung der betreffenden Gesichtshälfte aufgetreten, wie beispielsweise im Hammerschlagschen (3) Falle, wo er kurze Zeit nach einem mehr. stündigen forcierten Marsch in Schnee und Regen, bei dem die betreffende Gesichtshälfte besonders dem Sturm ausgesetzt war, sich einstellte; für diese Fälle wird von einigen Autoren Erkältung als Ursache der Erkrankung angenommen. Andere Male setzte die Erkrankung ein ohne erkennbare äußere Ursache; gleichzeitig bestehendes oder voraufgegangenes Fieber ließen auf eine infektiöse Ätiologie schließen. Eine solche ist auch für unseren Fall anzunehmen; auf eine Allgemeininfektion weist mit Sicherheit der Ikterus in Verbindung mit der erhöhten Temperatur hin. Die Eingangspforte des infektiösen Virus sind vielleicht die Gaumenmandeln, die bei der ersten Untersuchung stark gerötet gefunden wurden. Die Frage, welche Momente vorhanden sein müssen, damit bei einer Infektion des Körpers gerade die drei Hirnnerven alteriert werden, und zwar so, daB als Symptomkomplex der Erkrankung ein Herpes zoster oticus ausgelöst wird, soll hier nicht erörtert werden, da bakteriologische und pathologischanatomische Untersuchungen fehlen. DaB es sich nicht um eine zentrale Erkrankung, sondern um eine periphere Neuritis des $\mathrm{N}$. trigeminus, facialis und acusticus handelt, können wir mit Bestimmtheit annehmen. Die Erkrankung der drei Hirnnerven erfolgte nach Körners Beobachtungen in den von ihm als Herpes zoster oticus bezeichneten Fällen in bestimmter Reihenfolge hintereinander, primär war der Trigeminus affiziert, sekundär der Fazialis und Akustikus. Den Übergang der Neuritis aus dem Gebiete des Trigeminus auf den Fazialis und Akustikus erklärt Körner, der Anschauung Strübings (4) über das Zustandekommen einer Fazialislähmung bei Herpes zoster im Trigeminusgebiet folgend, durch die zahlreichen Anastomosen zwischen den Endästen des Trigeminus und Fazialis. Durch diese Anastomosen wird die Neuritis zentralwärts in den Fazialisstamm weiter geleitet und infolge einer toxischen Wirkung von Nerv zu Nerv durch die trennenden 
Gewebe hindurch ergreift sie den dem Fazialisstamm im Porus acusticus dicht angelagerten $\mathrm{N}$. acusticus.

Die Möglichkeit der primären Erkrankung des N. trigeminus und des Übergreifens der Entzündung von der Peripherie her auf dem erörterten Wege auf den Fazialis und Akustikus muß selbstverständlich zugegeben werden; eine Anzahl der publizierten Fälle macht es jedoch recht wahrscheinlich, daß die Ausbreitung des Entzündungsprozesses an den drei Hirnnerven in anderer Weise erfolgt. Mit genauer Krankengeschichte sind außer von Körner nur von Muck (5) (im Jahre 1912, Hammerschlag (3) (1898), Kaufmann (6) (1897) Fälle von Herpes zoster oticus veröffentlicht worden; letztere beiden Autoren gebrauchen zwar den erst später (1904) von Körner eingeführten Namen Herpes zoster oticus noch nicht, bezeichnen die Erkrankung als Neuritis des V., VII. ,VIII. Hirnnerven, ihre Fälle bieten aber genau den Symptomenkomplex des Herpes zoster oticus. Die Arbeiten Ramsays ( 7 u. 8) sind mir nur in einem kurzen Referat zugänglich gewesen, ich kann nicht angeben, ob die von ihm beobachteten 9 Fälle von Herpes zoster oticus ausführlich beschrieben sind.

Im Falle Mucks (5) erkrankte der Patient plötzlich gleichzeitig mit Schwindel, Sausen im Ohr, Schmerzen an der Stirnhälfte und hinter dem Ohr, schmerzhafter Schwellung und Rötung der Ohrmuschel, drei Tage später trat Fazialislähmung ein. Der Patient Hammerschlags (3) bekan zuerst Utbelkeit, starken Schwindel, wobei sich die Gegenstände von rechts nach links zu drehen schienen, tags darauf Ohrensausen und Ohrenstechen rechts, Brechreiz, Fazialislähmung, Schmerzen in rechter Gesichtshälfte, drei Tage später wurde Herpes zoster an der Ohrmuschel, dessen Entstehungstag nicht notiert ist, konstatiert. Bei Ka ufmanns (6) Kranken traten, nachdem fünf Tage lang Allgemeinerscheinungen - Unwohlfühlen, Mattigkeit, Kopfschmerz, Fieber bis $38,4^{\circ}$ bestanden hatten, an gleichem Tage Herpes zoster an der Wange, Schwindel und Erbrechen auf, vier Tage später Gesichtslähmung, Ohrensausen, Taubheit. Es haben also in diesen drei Fällen Symptome einer Erkrankung des N. acusticus, und zwar des Ramus vestibularis früher oder mindestens gleichzeitig mit Symptomen des $\mathrm{N}$. trigeminus bestanden, daher liegt es wohl nahe, zumal wenn die Erkrankung von Symptomen einer Allgemeininfektion begleitet ist, eine gleichzeitige Erkrankung der drei Hirnnerven an der Basis cranii 
anzunehmen. Man kann es sich aber auch vorstellen, da $B$ in diesen und manchen anderen Fällen die drei Hirnnerven nicht gleichzeitig, wohl aber in umgekehrter Reihenfolge, wie dies Körner annimmt, erkrankt sind, auch, daß die Neuritis nicht von der Peripherie zum Nervenstamm, sondern umgekehrt sich fortgepflanzt hat. Mangels anatomischer Befunde möchte ich ein derartiges Vorkommen nicht mit Sicherheit behaupten, aber doch wenigstens eine derartige Ansicht zur Erwägung stellen und begründen. Wenn in den erwähnten Fällen zu Beginn der Erkrankung nur über Symptome einer entzündlichen Alteration des Ramus vestibularis - Schwindel, Erbrechen - berichtet wird, so ist daraus meines Erachtens noch nicht zu schließen, daB Symptome von seiten des $R$. cochlearis nicht gleichzeitig bestanden haben und eine entzündliche Alteration dẹshalb nicht vorgelegen hat, denn bei keinem der Fälle wurde anfangs eine genaue Hörprüfung ausgeführt, und wir wissen ja zur Genüge, wie wenig dem Patienten oft recht beträchtliche Hörstörungen auf einem Ohre, zumal bei schwerer Störung des Allgemeinbefindens, zum Bewußtsein kommen. Bei der uns namentlich durch die neuere Literatur über die Neurorezidive bei Lues bekannt gewordenen großen Vulnerabilität des $\mathrm{N}$. acusticus gegenüber den übrigen Hirnnerven halte ich es jedenfalls für recht gut möglich, daß in den angeführten wie in manchen anderen Fällen der N. acusticus beim Herpes zoster oticus zuerst allein oder zusammen mit dem dicht benachbarten N. facialis im Stamm erkrankt war, daB der vom AkustikusFazialis-Stamm an der Schädelbasis räumlich relativ weit entfernt liegende $\mathbf{N}$. trigeminus im Stamm nicht ergriffen worden, sondern die partielle Erkrankung des N. trigeminus, die den Herpes zoster auslöst, durch Fortschreiten der Entzündung im N. facialis peripherwärts und Überleitung der Entzündung auf den N. auriculo-temporalis durch seine Anastomose mit dem $\mathrm{N}$. facialis entstanden ist. - Wenn die Lähmung des Fazialis immer erst mehrere Tage nach der Herpeseruption auftritt, so beweist dies ja nur, daB in diesem Moment die Entzündung erst so hochgradig wurde, daß alle Nervenfasern funktionsuntüchtig wurden, nicht, daß eine Entzündung leichteren Grades vorher nicht schon bestanden hat. Als Beispiel, daB ein Herpes zoster im Trigeminusgebiet auch nach dem Eintritt einer Fazialislähmung entstehen kann, führe ich einen bei Str übing (4) zitierten Fall Trydes an, wo ohne Beteiligung des $N$. acusticus nach längerem 
Bestehen einer Fazialislähmung ein Herpes zoster an der Stirn sich entwickelte. Unser Fall ähnelt in seinem Verlaufe sehr dem von Körner, in dem zunächst der Herpes an der Ohrmuschel auftrat und erst zwei Tage später die Fazialislähmung und Akustikusalteration manifest wurde. Trotzdem möchte ich vor allem wegen der sicher vorhandenen Allgemeininfektion aus den zuvor erörterten Gründen eher annehmen, daß die Erkrankung der drei Hirnnerven in den Nervenstämmen an der Hirnbasis begonnen und von da peripherwärts fortgeschritten ist als umgekehrt. Beide Fälle zeigen übrigens in der Beobachtung auch insofern eine Übereinstimmung, als beide Male eine ohrenspezialistische Untersuchung erst nach völliger Ausbildung des Symptomkomplexes eines Herpes zoster oticus stattfand, bei Körner 2 Wochen, bei uns 4 Wochen nach Erkrankungsbeginn.

Versuchen "wir von den in der Literatur ausführlicher mitgeteilten Fällen and dem unsrigen das Gemeinsame der klinischen Symptome und des Krankheitsverlaufes zusammenzufassen, so ergibt sich für den Herpes zoster oticus folgendes Krankheitsbild: „Bei vorher meist ganz gesunden Leuten, mit Vorliebe bei Männern im 3. bis 4. Jahrzehnt, tritt im Anschluß an eine einmalig zeitlich scharf begrenzte Erkältung oder an eine ohne bestimmte Veranlassung entstandene, mit Fieber verbundene, auf eine Allgemeininfektion hindeutende starke Störung des Allgemeinbefindens (Mattigkeit, Kopfschmerz, Appetitlosigkeit) durchsehnittlich 4-5 Tage später an der Ohrmuschel und ihrer Umgebung, eventuell auch im äußeren Gehörgang und am Trommelfell, eine Gruppe stecknadelkopf- bis linsengroßer, von entzündlich geröteter Haut umgebener, mit wasserklarem Inhalt gefüllter Bläschen auf - Herpes zoster —, dabei oder schon kurz zuvor werden manchmal reißende Schmerzen und ein Gefühl schmerzhafter Spannung in der betreffenden Gesichtshälfte empfunden. Entweder gleichzeitig oder kurz vor oder nach der Herpes-zoster-Eruption stellt sich unter Zunahme dex Kopfschmerzen starker Schwindel, hier und da Brechneigung ein, Ohrensausen und Ohrenstechen werden empfunden. Bereits am nächsten, längstens einige Tage nach der Herpeseruption, wird eine Lähmung des $\mathrm{N}$. facialis manifest, die sämtliche Gesichtsäste betrifft. Schwerhörigkeit kann schon beim Auftreten der Vestibularsymptome bestanden haben, wird aber meist vom Patienten erst einige Tage später bemerkt, wenn sie sehr hochgradig geworden oder Taubheit eingetreten 
ist. Geschmacksstörungen werden fast immer beobachtet, ihr Auftreten fällt zeitlich meist mit der Fazialislähmung zusammen. Der klinische Verlauf gestaltet sich dann só, daß die schweren Allgemeinerscheinungen in der Regel nach längstens 14 Tagen abklingen; das Schwindelgefühl und der Drehschwindel werden nach einigen Wochen geringer und verlieren sich nach spätestens 1-2 Monaten ganz, in welcher Zeit auch der Gang, der anfangs taumeind und von starkem Schwanken nach der erkrankten Seite begleitet ist, wieder normal wird. Das Verschwinden der Alterationserscheinungen des $R$. vestibularis und seiner Endorgane kann dadurch bedingt sein, daß der Nerv sich wieder erholt oder aber, daß der Vestibularteil des gesunden Ohrs alternierend eintritt, wenn der kranke Nerv völlig funktionsuntüchtig geworden ist. DaB beides - Rückbildung der Entzündungserscheinungen am $\mathrm{N}$. vestibularis und zum Funktionsausfall des Vestibularapparates führende Schädigung desselben - eintreten kann, wissen wir aus den beiden seit Einführung der Vestibularprüfung in die otologischen Untersuchungsmethoden beobachteten Fällen, dem von Muck und dem unsrigen. In beiden Fällen war auf der Höhe der Erkrankung kalorischer Nystagmus nicht auslösbar, bei Muck stellte er sich nach vier Wochen wieder ein, in unserem Falle blieb der Vestibularis dauernd kalorisch und für Drehreiz unerregbar. Von Kochlearissymptomen halten Ohrensausen, Diplakusis eventuell monatelang an, Rückkehr des Hörvermögens zur Norm ist selten, meist bleibt es dauernd so geschädigt, daß laute Sprache nur auf einige Meter Entfernung noch gehört wird. Es ist beobachtet, daß das Hörvermögen allmählich im Laufe der nächsten Monate schlechter wird als bei voll ausgebildetem Symptomkomplex des Herpes zoster oticus; Taubheit kann, wie unser Fall zeigt, von Anfang an unverändert bestehen bleiben. Die Fazialislähmung bessert sich auch in den schwersten Fällen wohl stets, aber dazu braucht es anscheinend oft recht lange Zeit, ein Jahr und darüber, nur selten tritt aber eine völlige Restitutio ad integrum ein, und leichte Paresen in einem oder mehreren Gesichtsästen bleiben oft dauernd bestehen. Geschmacksstörungen auf der Zungenhälfte der kranken Seite werdon beobachtet. - Die Diagnose wird kaum in einem Falle, sobald der gesamte Symptomkomplex ausgebildet ist, Schwierigkeiten machen. Es ist notwendig, wenn ein Herpes der Ohrmuschel als erstes Symptom auftritt, sogleich eine genaue Hörprüfung und Vestibularprüfung zu 
machen, weil ein Funktionsausfall in dieser Richtung gleich darauf hinweist, daß es sich nicht um einen einfachen Herpes im Trigeminusgebiet handelt. Kommt der Kranke nach Abheilung des Herpes zoster erst zur Behandlung und sind nur noch die Symptome der Fazialis- und Akustikusneuritis manifest, so wird man nach eventuellen Residuen des Herpes - Narben - genau fahnden, auch anamnestisch in dieser Richtung sorgfältig eruieren müssen. Daß man in solchen Fällen zur Sicherstellung der Diagnose andere Erkrankungen. welche zu Alterationen des Kochlear- und Vestibularteiles von $\mathrm{N}$. acusticus sowie des $\mathrm{N}$. facialis führen, insbcsondere Erkrankungen des Mittelohres und Labyrinths, ausschließen muß, jedenfalls auch immer die Wassermannsche Reaktion anstellen muß, ist selbstverständlich.

Die Prognose des Herpes zoster oticus ist nach dem Gesagten bezüglich Restitutio ad integrum jedenfalls nicht durchaus günstig, da völlige Heilungen selten sind; Besserungen des Gehörs und der Fazialislähmung sind aber in den meisten Fällen zu erwarten, die Vestibularsymptome schwinden stets, auch bei kompletter dauernder Funktionsuntüchtigkeit des erkrankten $\mathrm{N}$. vestibularis.

Therapeutisch sind beim Herpes zoster oticus, abgesehen von der üblichen lokalen Salben- und Puderbehandlung des Herpes, namentlich bei Erkältungsätiologie, innerlich Antirheumatika angewandt worden, auf der Höhe der Erkrankung dabei Narkotika, ferner Jodnatrium, laue Bäder, gegen Hörstörungen Injektionskuren mit Pilokarpin, zur Behebung der Fazialislähmung Faradisation und Galvanisation. Daß man die elektrische Behandlung jedenfalls nicht zu früh abbrechen soll, ergibt sich daraus, da B z. B. in unserem Falle trotz nur geringer Besserung der Lähmung nach einem halben Jahr keinerlei elektrische Entartungsreaktion am Fazialis bestand. Poljtzer meint, daB der Ausgang des Krankheitsprozesses von der Therapie unabhängig sei.

\section{Literatur.}

1. Körner, ,Über den Herpes zoster oticus" (Herpes an der Ohrmuschel mit Lähmung der A. acusticus und des N. facialis). Münchn. Med. Wochenschr. 1904, Nr. 1.

2. Lesser, "Verhandlungen des IV. Dermatologenkongresses".

3. Hammexshlag, ,Beitrag zur Kasuistik der multiplen Hirnnerverkrankungen". Arch. f. Ohr. Bd. $45, \mathrm{~S} .1$.

4. Strübing, ,Deutsches Archiv für klinische Medizin", Bd. 37. 
5. Muck, ,Neuritis des N. trigeminus, facialis und acusticus als Symptomkomplex eines. Herpes zoster ${ }^{6}$. Zeitschr. f. O. Bd. 64, S. 217.

6. Kaufmann, „Utber einen Fall von gleichzeitiger akut aufgetretener Erkrankung des Akustikus, Fazialis und Trigeminus". Zeitschrift f. O. Bd. 30, S. 125 .

7. Ramsay-Hunt, J., New York, ,Der Symptomkomplex der akuten Poliomyelitis des Ganglion geniculi, oticum, glossopharyngeum und pneumogastricum. Archives of intern. med. Vol. 5, 15. Juni. Ref. Z. f. O. Bd. 63 , S. 239 .

8. Ra msay-Hunt, J., New York, ,Über den sensiblen Symptomkomplex im Gebiete des N. facialis". Archives internation. de Laryngologie 1910, Nr. 1. Ref. Z. f. O. Bd. 62, S. 95.

9. Körner, ,Lehrbuch der Ohrenheilkunde"

10. Bönninghaus, ,Lehrbuch der Ohrenheilkunde", S. 312.

11. Politzer, „Lehrbuch der Ohrenheilkunde", S. 202, S. 611.

12. Sarai, ,Herpes der Ohrmuschel mit Neuritis des N. facialis“. Z. f. O. Bd. 46 , S. 136 . 